\title{
Determination of Imbalance Problem in Electric Motor and Centrifugal Pump by Vibration Analysis
}

\author{
R. KILIÇ* \\ SASKİ General Directorate, Kentpark İçi, PK 144, Sakarya
}

\begin{abstract}
In this study, general characteristics of imbalance problem in electric motor and centrifugal pumps and the vibrations caused by these faults are discussed. The fact that frequencies would yield vibrations which were caused by imbalance problems was investigated in detail. In addition, the vibration behavior formed by the electric motor and centrifugal pump running in water pumping mode are presented as a sample case. It is observed that the imbalance problem which have occurred in the electric motor and centrifugal pump could be easily determined using the vibration analysis.
\end{abstract}

DOI: 10.12693/APhysPolA.130.487

PACS/topics: 43.40

\section{Introduction}

Solution of vibration problems in the electric motors and centrifugal pumps is important for proper operation and performance. Excessive vibrations seen in the electric motors and centrifugal pumps shorten the life of the machine and lead to the mechanical fatigue and even to damage of the machine. The causes of these vibrations in the electric motors and centrifugal pumps may be mechanical, fluid-borne or electromechanical [1]. Many of the mechanical and electromechanical-borne vibrations are directly related to the machine speed and can be determined by vibration analysis [2]. It is necessary to carry out the measurements in order to determine the source of vibrations. The source of the vibration can be determined by examining the relationships among data obtained from these measurements in the time and frequency domains.

Imbalance is one of the vibration sources in the electric motors and centrifugal pumps. The main causes of this problem in the electric motors and the pumps are: defects during manufacturing, corrosion in the pump fan, partial permanent pollution of the pump fan blade, the unbalanced mounting of one of the pump bearings carrying the whole loads of pump fan, improper mass distribution in the rotor arms and blades of the electric motor, the bending of the pump and electric motor shaft. The imbalance problem encountered in electric motor and centrifugal pumps causes negative effects on pump efficiency [3]. In this study, the vibrations of an electric motor and centrifugal pump were evaluated in the time and frequency domain. As a result of this evaluation, the direction and the frequencies, at which the peaks of vibration were formed by imbalance, was obtained in the frequency domain and the effects of these peak values in the time domain were plotted.

*e-mail: recep.kilic@sakarya-saski.gov.tr

\section{Identification of imbalance problem}

The state of equilibrium of all forces formed by the rotating machine elements is called equilibrium (balance). Any change in this state of equilibrium creates imbalance. Imbalance is the most common source of vibration seen in machines [4].

Imbalance is a linear problem. If a rotor is unbalanced, it will have the same amount of imbalance throughout the cycle of 360 degrees. Each cycle in the time domain will have the same amplitude, at the same time, the image of the time signal will have a sinusoidal structure [5]. Imbalance may appear in two ways: static and dynamic. In case of static imbalance, centrifugal force is applied on bearings at $1 X$ frequency. The $1 X$ force here is directly proportional to the square of rotor speed. Imbalance forces in electric motors and centrifugal pumps always cause vibration in the manner that there will be 90 degrees of phase difference between bearings, tangential and radial $1 X$ components $[6]$. This is the definite test of imbalance and it is used for determination of imbalance due to misalignment and in analysis of the vibration plots.

In the vibration analysis, imbalance always creates high vibration amplitudes at $1 X$ RPM. If imbalance is severe, it manifests itself in the frequency spectrum along with imbalance at operating speed. These frequencies can sometimes make it difficult to test the imbalance, however, the existence of the imbalance cannot be mentioned without any evidence of vibration amplitude at $1 X \mathrm{RPM}$ frequency. If there is $1 X \mathrm{RPM}$ component in the frequency spectrum, imbalance will be at the beginning of the list of probable causes [5].

While analyzing the vibration spectra, vibration peaks showing imbalance in the radial and tangential direction should be compared. Vibration peaks in the radial direction will have larger amplitude than the vibration peaks in the tangential direction. The severity of the imbalance will be indicated by the closeness of these peaks in both directions, which are the imbalance indicators, to each other. Vibration peaks in the axial direction will have extremely low amplitude [7]. 
Attention should be paid to this topic in the analysis of vibration of the motor pumps which are vertically mounted. Since the back part of engine is not mounted on anything, the $1 X$ vibration occurs in the engine. While determining the motor imbalance, $1 X$ measurements should be made by firstly removing the coupling and starting the motor alone, in order to isolate the imbalance of the motor from the imbalance of the pump. In this case, the problem is in motor if $1 X$ levels of free side of the engine are high. Otherwise the problem is in the pump [8]. The discussed features of the imbalance measurement are summarized in Table I.

TABLE I

Imbalance malfunctions dashboard.

\begin{tabular}{l}
\hline \hline Dominant vibration frequency \\
\hline$\bullet$ Vibration peaks at radial and tangential \\
$1 X \mathrm{RPM}$, vibration peaks with low ampli- \\
tude and low axial vibration at $2 X \mathrm{RPM}$ \\
and $3 X \mathrm{RPM}$. \\
$\bullet$ The time signal has a sinusoidal form. \\
$\bullet 1 X \mathrm{RPM}$ peaks should occur in the same \\
direction on both bearings. \\
$\bullet 1 X \mathrm{RPM}$ peaks in the radial direction are \\
greater than the $1 X \mathrm{RPM}$ peaks in the tan- \\
gential direction \\
$\bullet$ Approximate imbalance values: \\
$1 X \mathrm{RPM}$ radial $>4 \mathrm{~mm} / \mathrm{s}$ \\
$1 X \mathrm{RPM}$ tangential $>3 \mathrm{~mm} / \mathrm{s}$ \\
Low axial value. \\
If the ratio between radial and tangential is \\
greater than 0.25 and smaller than 4, there \\
is an imbalance.
\end{tabular}

Tangential

Axial

\section{Materials and methods}

PREDICT-DLI DCA-20 data collector program for the determination of the imbalance problem in the electric motors and centrifugal pumps and ExpertALERT for Voyager programs were used in this study. PREDICTDLI DCA-20 data collector system consists of two parts, hardware central processing unit and the software.

By using PREDICT-DLI DCA-20 data collector system, one or more measurement points can be defined on each electric motor and centrifugal pump. PREDICTDLI DCA-20 data collector can make measurements along 3 axes or only one axis, the point to be measured can be easily identified using a barcode. Measurement can be made in the range of $40-60 \mathrm{kHz}$ and a 6400 -line spectrum can be taken.

ExpertALERT for Voyager program which was used in the study is composed of two components. The first component is the program file, and the second section is the database where facility information, motor-pump information, the results and report information obtained from measurement are stored. All features of the electric motor and centrifugal pumps (speed, being horizontal or vertical, the drive unit, etc.) can be introduced separately to the program.

In our study carried out to determine the imbalance problem in electric motors and centrifugal pumps with vibration analysis, first the vibration measurement points and measurement directions were determined. Determination of measurement points before making vibration analysis of electric motors and centrifugal pumps helps to determine the best data collection points for the analysis and to define the potential sources of the vibration [9]. For this purpose, the closest points to the bearings in electric motors and centrifugal pumps were selected as measurement points. While selecting these points, areas of metal to metal joints, painted surfaces, unloaded bearing areas, split and cracked bearings and the structural spaces were avoided.

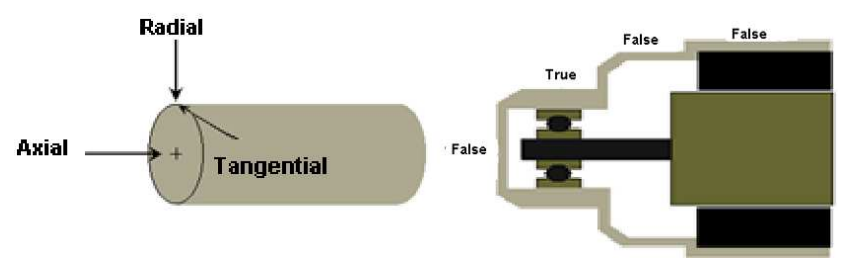

Fig. 1. Schematic view and measurement directions of the accurate measurement points near ball bearings.

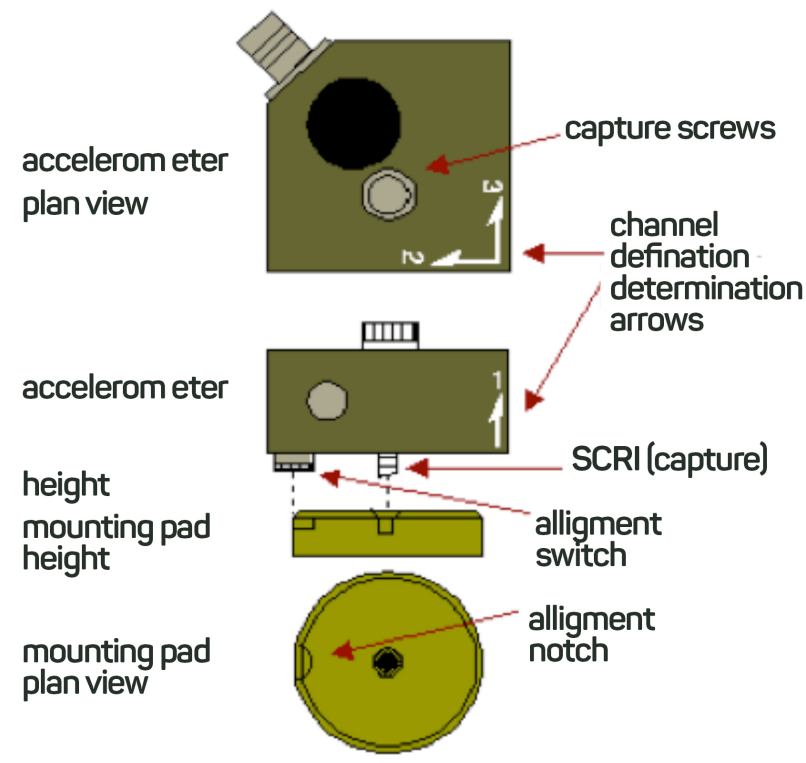

Fig. 2. Acceleration detector and mounting pad used in vibration measurement.

Taking the vibration measurements always in same point and directions is very important in terms of storing the vibration data obtained from the electric motors and centrifugal pumps for comparative purposes and for monitoring the trend curves [10]. The acceleration detector and the assembly block are shown in detail in Fig. 2. Detector sensors were designed to be oriented always in 
the same direction. The channels marked with numbers of 1,2 and 3 on the detector can be allocated to different measurement axes according to the states of the electric motors and centrifugal pumps. If the detector is mounted on the ball bearing in a horizontal motor-pump, channel 1 will be radial ( $\mathrm{R})$, channel 2 will be tangential $(\mathrm{T})$, perpendicular to the shaft axis and channel 3 will be axial (A), parallel to the shaft axis. This situation is called RTA. Other designations (RAT, ATR, TRA) are performed according to the measurement point of the system.

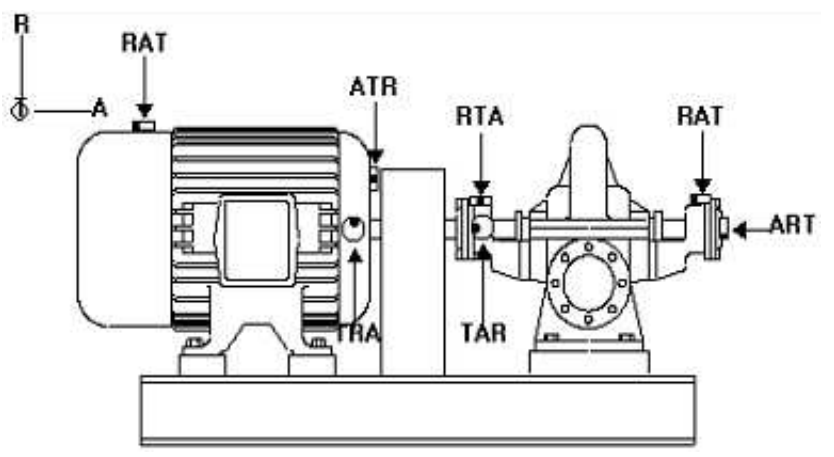

Fig. 3. Different measurement directions that can be taken on a pump.

In the study, primarily, all adjustments of the electric motors and centrifugal pumps were made in the best way to reduce the levels of vibration under $1 \mathrm{~mm} / \mathrm{sec}$. Then the vibration measurement points mentioned above were determined and vibration measurement mounting blocks were glued to the four points identified on electric motor and centrifugal pump, with the aim to ensure continual measurement of vibrations from the same point. In Fig. 3, the measurement locations and directions of vibration mounting blocks, placed on motor-pump, are shown. Table II lists the technical features of the electric motors and centrifugal pumps on which the vibration measurements were made and the imbalance was determined in the study.

TABLE II

Technical features of the studied electric motors and centrifugal pumps.

\begin{tabular}{c|c|c}
\hline \hline & Motor & Pump \\
\hline Brand & SIEMENS & ABS SCANPUMP \\
Power & $560 \mathrm{~kW}$ & $560 \mathrm{~kW}$ \\
Cycle & $1480 \mathrm{c} / \mathrm{min}$ & $1480 \mathrm{c} / \mathrm{min}$ \\
Number of blades & & 6 \\
Capacity & \multicolumn{2}{|c}{ Flexible coupling } \\
Pump head & NU 222 & $656 \mathrm{~m}^{3} / \mathrm{h}$ \\
Coupling type & NU224/6222 C3 & 7317 \\
Free side & \multicolumn{2}{|c}{$\mid$}
\end{tabular}

\section{Findings and discussion}

In pump lift stations (PLS), the imbalance problem encountered in electric motors and centrifugal pumps causes negative effects on pump efficiency. Many imbalance problems were observed in our study. Study was carried out in order for the problem not to cause secondary malfunctions in PLS. Plots of vibrations caused by the pump fan imbalance of the electric motors and centrifugal pumps are analyzed below in detail.

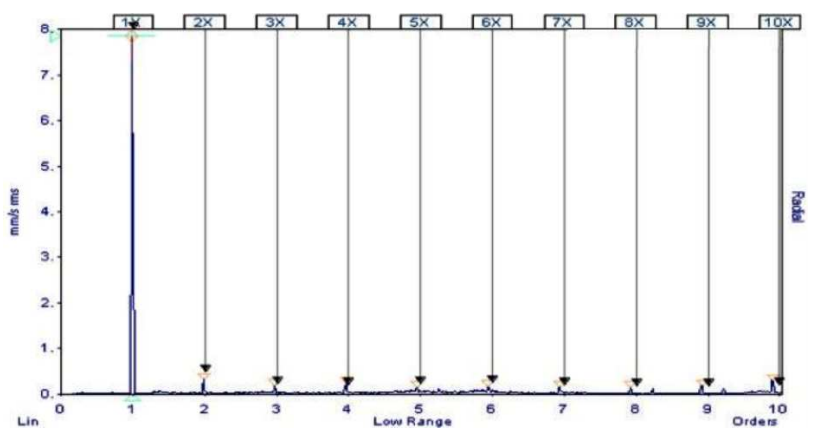

Fig. 4. Pump coupling side radial direction spectrum.

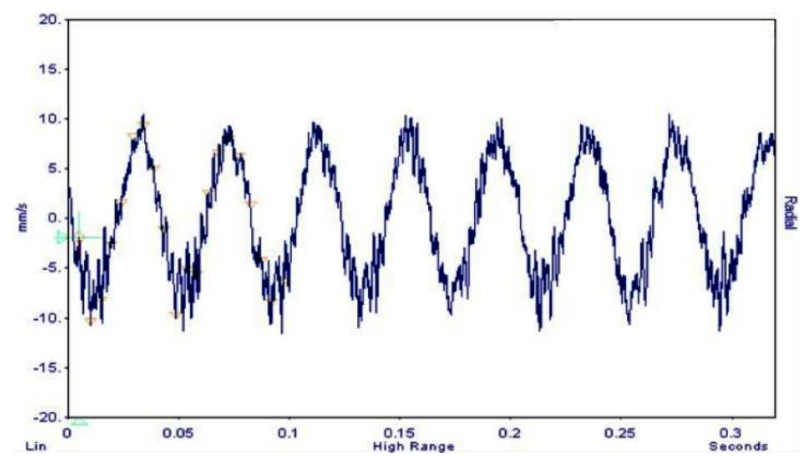

Fig. 5. Pump coupling side radial direction waveform.

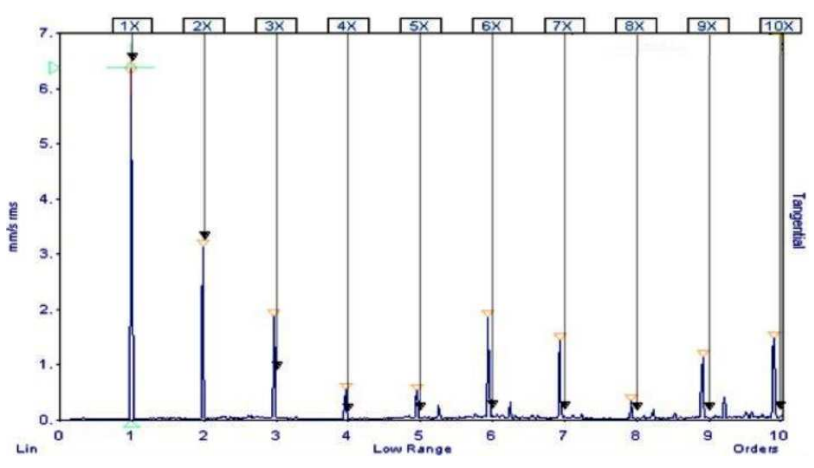

Fig. 6. Pump coupling side tangential direction spectrum.

Vibration measurements were performed in certain periods during motor-pump operation. As it can be seen in the plots, an increase of up to $100 \%$ was observed in the vibration amplitudes in the latest measurements.

When vibration measurements are analyzed in detail, the amplitudes of radial and tangential vibrations at 
$1 X$ RPM of motor speed (Figs. 4, 6, 8 and 10) lead us to suspicions that there might be an imbalance problem. The mechanical space and the coupling misalignment produce vibrations at the same frequency.

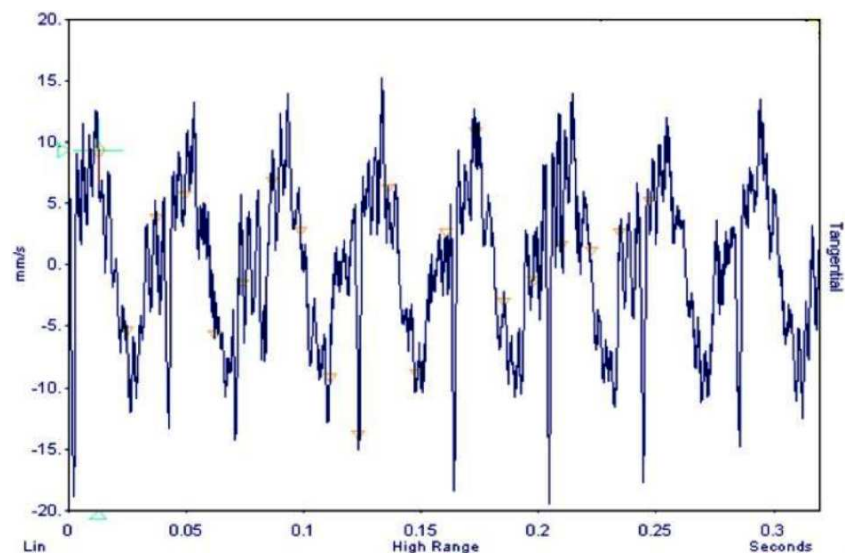

Fig. 7. Pump coupling side tangential direction waveform.

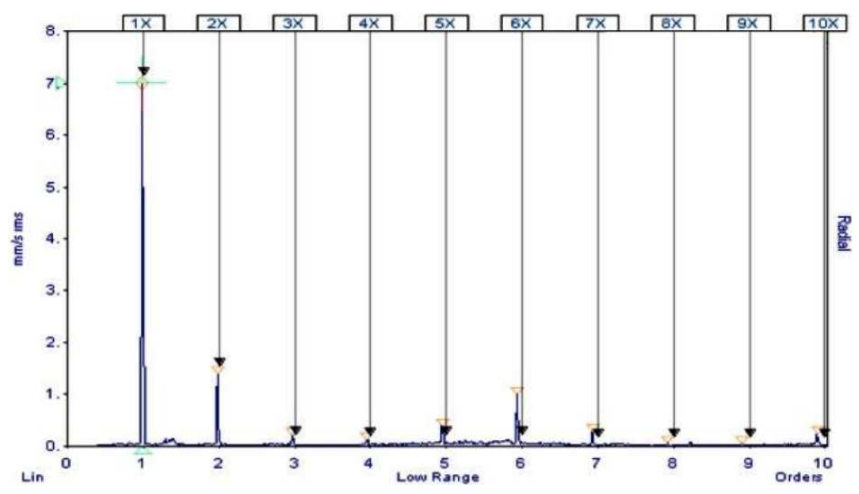

Fig. 8. Pump impeller side radial direction spectrum.

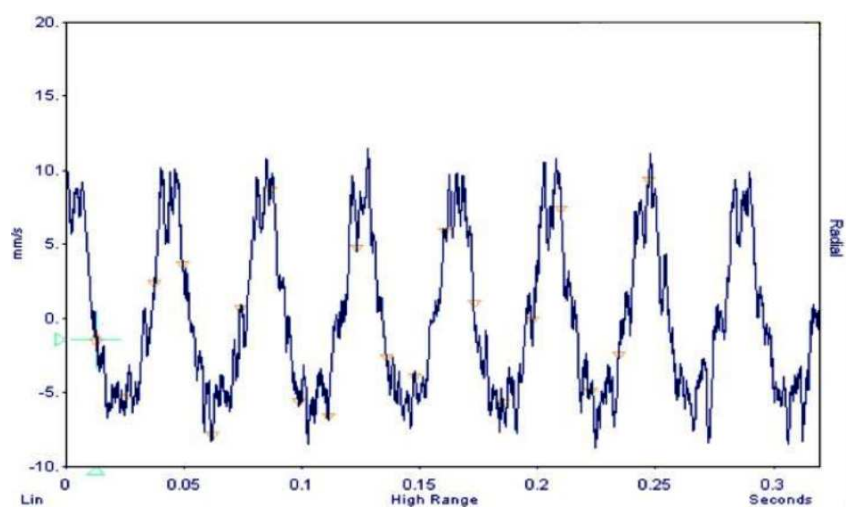

Fig. 9. Pump coupling side radial direction waveform.

When looking at the spectra of the vibration measurements taken from both free side of the pump (Figs. 8 and 10) and coupling side of the pump (Figs. 4 and 6), the amplitude value at $1 X$ RPM frequency in the vibration plots in radial direction is greater than the amplitude value at $1 X \mathrm{RPM}$ frequency in tangential direction,

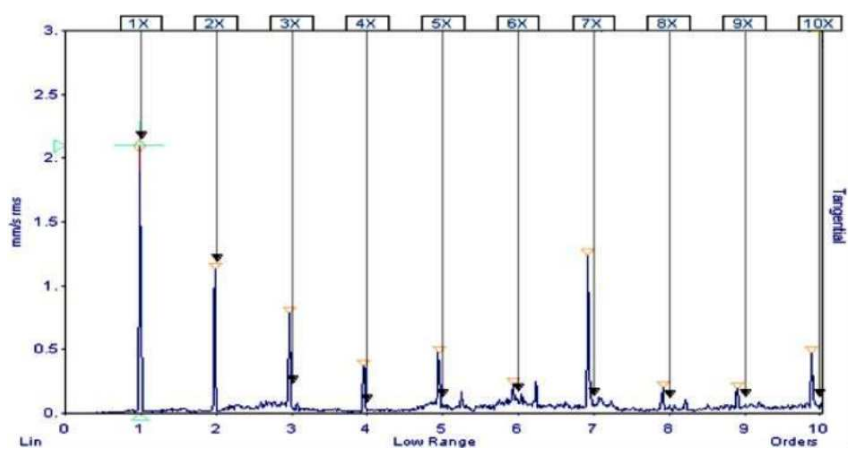

Fig. 10. Pump impeller side tangential direction spectrum.

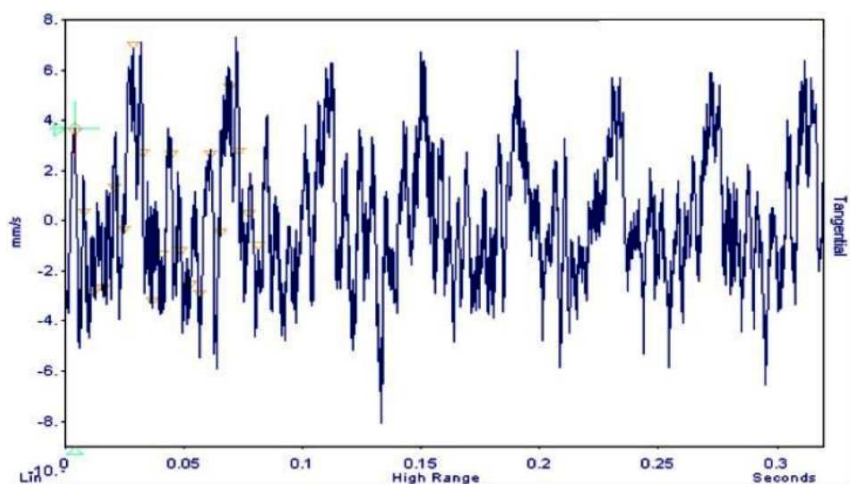

Fig. 11. Pump impeller side tangential direction waveform.

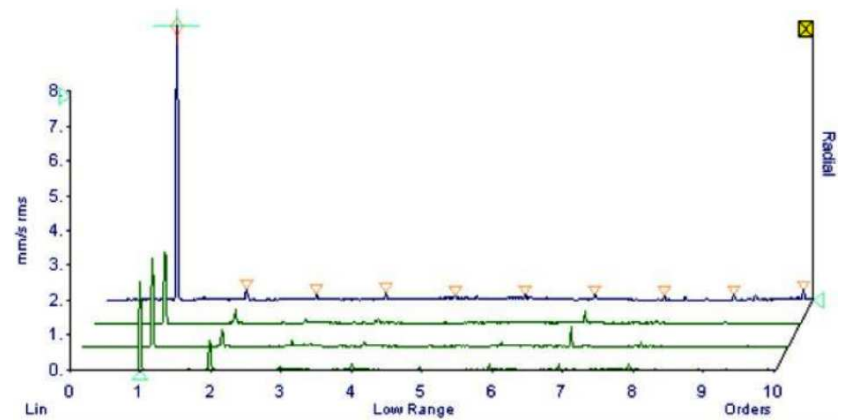

Fig. 12. Comparison of pump coupling side radial direction spectra.

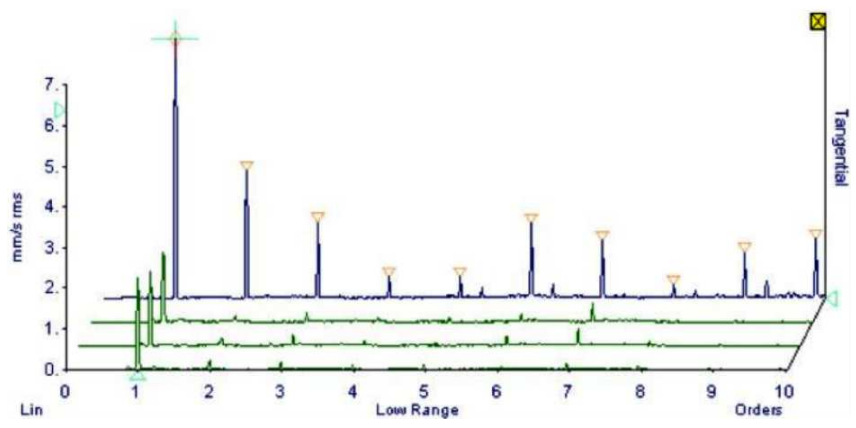

Fig. 13. Comparison of pump coupling side radial direction spectra. 


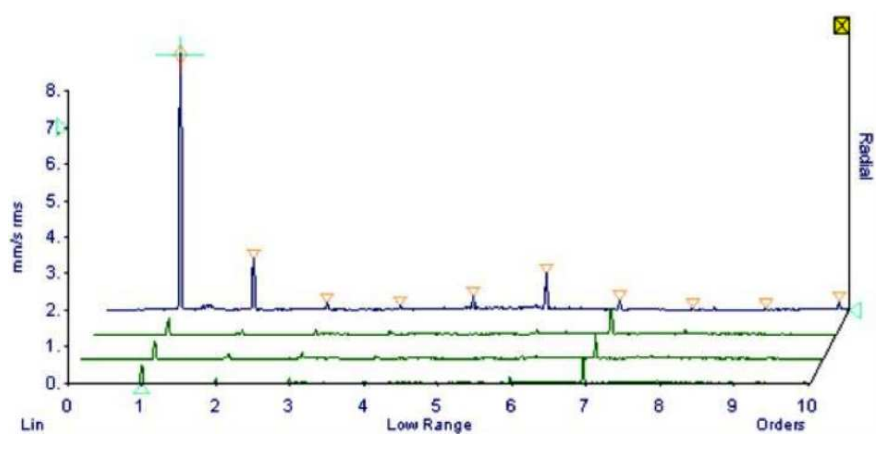

Fig. 14. Comparison of pump impeller side radial direction spectra.

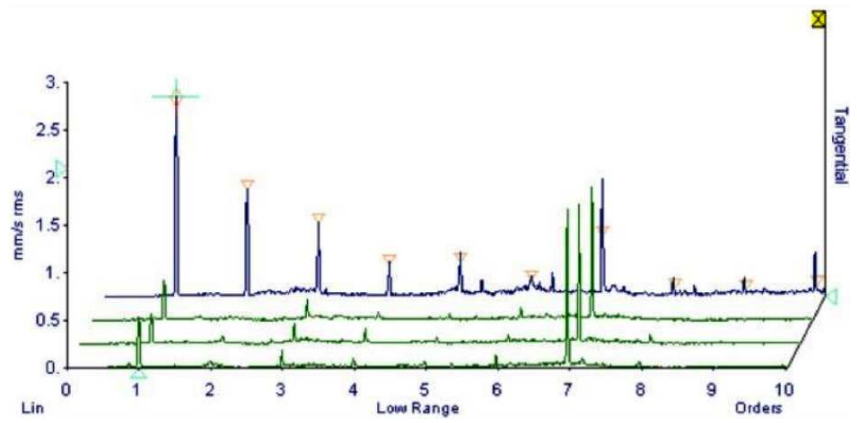

Fig. 15. Comparison of pump impeller side tangential direction spectra.

which increases the likelihood of imbalance. Moreover the measurements of amplitude values in the radial direction taken from both sides (Figs. 4 and 8) are greater than $4 \mathrm{~mm} / \mathrm{s}$, which reinforces this possibility. In this case, vibration plots should be examined in the frequency and time domains. When looking at the vibration plots of pump free side and coupling side in the time domain, the signal has a sinusoidal structure (Figs. 5, 7, 9 and 11). This situation is exactly the evidence of the imbalance problem.

In Figs. 12-15, the changes of the vibration amplitudes in the radial and tangential direction of both pump free side and pump coupling side can be observed during the occurrence of malfunction.

After this discovery, the pump was stopped and the necessary intervention was performed. When the pump was opened, it was observed that two of the fan blades were corroded. It was seen that this imbalance was caused by the corrosion on pump blades.

\section{Conclusions}

Imbalance is one of the major problems encountered in the electric motors and centrifugal pumps. In order to discover the imbalance by the vibration analysis, the following points should be noted.
- The points to be measured on the system must be determined accurately.

- Reference measurement points should not be changed.

- While detecting the imbalance by vibration analysis, results should be viewed in both, the time and vibration frequency domains.

- According to the importance of the measured system, the vibration analysis should be done using determined reference values.

- Permanent measurement points must be determined for critical electric motors and centrifugal pumps. Online measurement must be made at this points and it must be saved to the database. The recorded data must be evaluated by the software to do the necessary analysis.

\section{References}

[1] N. Aktürk, R. Gohar, Proc. Instn. Mech. Engrs. 212, 101 (1998).

[2] J. Piotrowski, Why Shaft Misalignment Continues To Befuddle And Undermine Even The Best CBM And Pro-Active Maintenance Programs, www. maintenanceresources.com, 2001.

[3] B. Leimkuhler, Vibration Monitoring of Electric Motor Bearing, SKF Condition Monitoring, Inc. Technical Paper CM1007, 1999.

[4] S. Orhan, Teknoloji 3, 41 (2003).

[5] W. Victor, Machinery Vibration Measurement and Analysis, McGraw Hill Book Company, 1991.

[6] J.I. Taylor, The Vibration Analysis Handbook, Vibration Consultants, 1994.

[7] D.W. Gelen, Introduction To Machine Vibration, DLI Engineering Corp., 1993.

[8] R.L. Eshleman, Machinery Vibration Analysis II, Vipress, 1996.

[9] S.R. Bognatz, Alignment of Critical and Noncritical Machines, www. bently.com, 2000

[10] D. Yu, J. Cheng, Y. Yang, Mech. Syst. Signal Proc. 19, 259 (2005) 